\title{
Building Blocks for Integrated Graphene Circuits
}

\author{
Denis A. Areshkin*,† and Carter T. White ${ }^{\ddagger}$ \\ George Washington University, Washington, DC 20052, Naval Research Laboratory, \\ Washington, DC 20375
}

Received March 26, 2007; Revised Manuscript Received May 30, 2007

\begin{abstract}
The observation of single sheets of graphite (graphene) presents new possibilities for carbon-based nanoelectronics. We report defect tolerant configurations for a nearly reflectionless $120^{\circ}$ turn and nearly reflectionless symmetric and asymmetric splitters, which can be cut from graphene. Connections between zigzag strips of different widths can be made with either low or high reflectance depending on the connection shape.
\end{abstract}

The recent observation of isolated graphite sheets (graphene) supported by insulating substrates ${ }^{1-4}$ provides new possibilities for carbon-based nanoelectronics. The potential importance of graphene as an electronic material is partially based upon the following properties: (1) Easy atomic plane lamination and strong bonding within the plane make graphene fabrication technologically accessible through both mechanical exfoliation ${ }^{2,3}$ and epitaxial growth. ${ }^{4}$ (2) Devices and integrated circuits (ICs) could be processed from a graphene sheet using the existing planar technologies: electron- or ion-beam lithography ${ }^{5-7}$ or direct focused electron $^{8}$ or ion-beam ${ }^{9}$ writing. The latter two could be very precise because of the low electron or ion flux required for carving a single atomic plane, which decreases the Coulomb repulsion between particles in the beam and hence allows superior beam focusing. (3) The main building blocks of a graphene IC are graphene nanostrips (GNSs) 5-10 nm wide, which can have properties ranging from metallic to semiconducting depending on their widths and edges. ${ }^{10}$ (4) Zigzag-edge GNSs have very low sensitivity to edge and substrate-induced disorder ${ }^{11}$ and can transmit currents of an order of tens of microamps for voltage ranges $0.5-1.0 \mathrm{~V}$ in the absence of spin polarization along the edges. (5) In the single-channel regime, the ballistic length of GNSs can be comparable to the ballistic length of carbon nanotubes in the presence of substrate-induced disorder. ${ }^{11}$ (6) Low scattering in the single-channel regime, including suppression of acoustic phonon scattering, ${ }^{12}$ allows high current densities through GNSs with low heat dissipation, making them promising materials for Terahertz ICs.

The above properties have already stimulated research on nanostrips fabrication ${ }^{4,13,14}$ and will most likely result in

\footnotetext{
* Corresponding author. E-mail: denis.areshkin@nrl.navy.mil.

George Washington University.

$\doteqdot$ Naval Research Laboratory.
}

attempts to create all-graphene electronic nanodevices and circuits patterned from either a single graphene plane or multiple planes separated by layers of insulating material. Either circuit concept requires passive elements and interconnects for wiring individual circuit elements. The wiring turns out to be a nontrivial issue because as we shall see only a few specific GNS patterns that have nearly ideal conductance and can transmit electron flux without losses. Most other patterns possess substantial reflectivity and cannot be used for wiring purposes despite their metallic nature. In this paper, we suggest geometrical configurations for (1) reflectionless interconnects and (2) highly reflective patterns analogous to resistors.

Perhaps it is easiest to motivate these patterns by speculating about a possible configuration of a simple graphene IC, e.g., the flip-flop circuit for high-frequency signal generation depicted in Figure 1. To connect the electronic components one needs to transmit, split, and turn the electron flux with minimal losses. Our simulation results indicate that the 50/ 50 splitter $\mathrm{S} 1$, and $120^{\circ}$ turn $\mathrm{T} 1$ depicted in Figure $1 \mathrm{~b}$ possess nearly zero reflection, while the reflection of most other configurations is comparable to their transmission. Serial connections of highly reflective $60^{\circ}$ turns marked as R1R4 serve for controllable current attenuation. Zigzag-edge GNSs, rather than armchair-edge GNSs, are preferred as interconnecting wires because they show a higher tolerance to edge imperfections. ${ }^{11}$ For that reason, we align the conjectured circuit in Figure $1 \mathrm{~b}$ in such way that all interconnecting wires align with $[1,0]$ crystallographic directions.

The design of the active components cannot be directly checked with the non-self-consistent tight-binding approach used herein, which does not allow calculation of the voltage drop needed to simulate the transistor operation. However, even this model can provide some guidance for a potential 


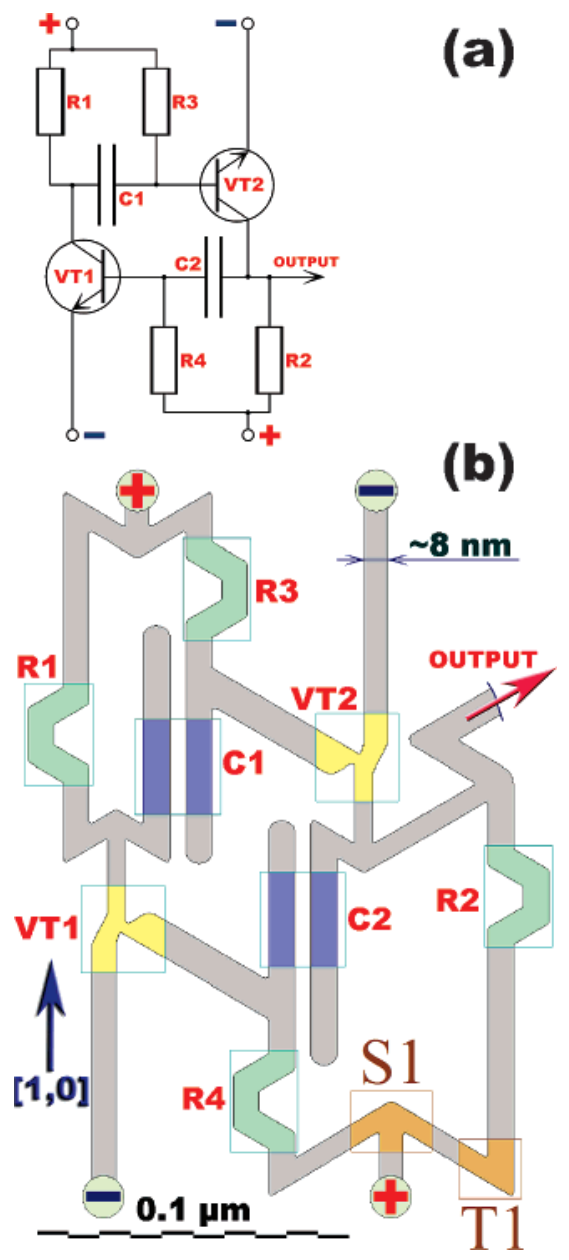

Figure 1. (a) Flip-flop circuit without wire crossings. (b) Its possible GNS-based counterpart patterned from a single graphene sheet. To keep this drawing compact, the capacitors $\mathrm{C} 1$ and $\mathrm{C} 2$ are not drawn to scale.

transistor design relying only on a single sheet. The simplest configuration of these postulated single-plane transistors (VT1 and VT2 in Figure 1b) is a zigzag-edge metallic channel with a short armchair-edge region incorporated between the source and the drain. Source and drain strips are of different widths, e.g., 8 and $7 \mathrm{~nm}$. The short armchair segment with variable width connects the source and the drain strips. Because of the width variation, a semiconducting region is expected to be present in the armchair segment. The role of this region is analogous to the base in a bipolar transistor. The wider control zigzag-edge GNS (e.g., $15 \mathrm{~nm}$ ) connected to the armchair segment serves as the base lead. At the point of connection to the armchair segment, the width of the base strip is abruptly decreased. This constriction results in a strong reflection of the electrons traveling in the base strip toward the channel. The non-self-consistent simulations indicate that only $\sim 1 \%$ of electrons penetrate from the base lead into the channel. The constriction effectively isolates the control lead from the channel. The increase of the gate potential is assumed to shift the band gap in the armchair region above the Fermi level in the drain and allow electron transmission through the source-drain channel. Although an ON/OFF ratio of such a single-plane transistor will be insufficient for digital circuits, it might provide stable signal generation for the flip-flop circuit envisaged in Figure 1b.

The rest of the paper is organized as follows. First, we discuss the model used, including the applicability of a spinrestricted approach to transport simulations. This issue is important because, according to spin-unrestricted Hubbard model and DFT simulations, the ground state of zigzag-edge GNS corresponds to a spin-polarized solution. ${ }^{10,15,16}$ At the same time, our simulation results indicate that the spin polarization in GNSs is destroyed under certain nonequilibrium conditions, leading us to believe that the spin-restricted model is more appropriate to transport simulations. Next, we explain the reason for insensitivity of ballistic conductance in zigzag GNSs to edge defects and discuss operational requirements for GNS-based circuits. Then we discuss ballistic transport through symmetric and asymmetric splitters and a $120^{\circ}$ turn. Transitions between zigzag-edge GNSs of different widths along with a $60^{\circ}$ turn and a combination of $60^{\circ}$ turns are treated next. Smooth transitions are almost reflectionless, while abrupt transitions can introduce up to $\sim 70 \%$ reflection and can serve as resistor analogues.

The results of this paper were obtained by using a $\pi$-orbital tight-binding model with interactions included up to second nearest neighbors, with first $\left(V_{1}\right)$ and the second neighbor $\left(V_{2}\right)$ parameters taken as -2.7 and $-0.069 \mathrm{eV}$, respectively. Longer range one-electron interactions are known to be important in describing armchair-edge strips but are neglected because they have little effect on the electronic structure of zigzag-edge GNSs in the region of primary interest. ${ }^{17}$ Indeed, the second-nearest-neighbor interaction is included only to bend otherwise essentially flat bands slightly downward (see Figure 2a), making the computations easier.

This model also neglects spin polarization effects at the zigzag-edge GNS edges. However, Fujita et al. have shown that, because of the nearly flat bands arising in a spinrestricted calculation (cf. Figure 2a), the edges of these strips should become spin polarized with ferromagnetic coupling between the major magnetic moments formed from itinerant electrons along each edge and antiferromagnetic coupling between these moments across the strip. ${ }^{10} \mathrm{~A}$ similar picture emerges in first-principles LDF calculations. ${ }^{15,16}$ However, this spin-polarized ground state can become unstable with respect to the spin-unpolarized state in the presence of a ballistic current through the strip. Indeed, we find by using Fujita and co-worker's model and their self-consistent approach ${ }^{10}$ that even if the Hubbard $U$, which favors spin polarization, is as large as $\left|V_{1}\right|$, the spin-polarized state is unstable with respect to the spin-unpolarized state if the minimum applied voltage bias exceeds about $0.4 \mathrm{~V}$. Furthermore, when the applied bias is removed, a spin redistribution must take place for the system to return to the spin-polarized ground state with electrons of the same spin accumulating along the corresponding edges. Because there is little overlap between the states belonging to different edges, this spin redistribution probably proceeds via processes where an electron scatters from one edge into a state associated with the strip interior at similar energy and then scatters to the opposite edge. The rate of such scattering is expected to be 


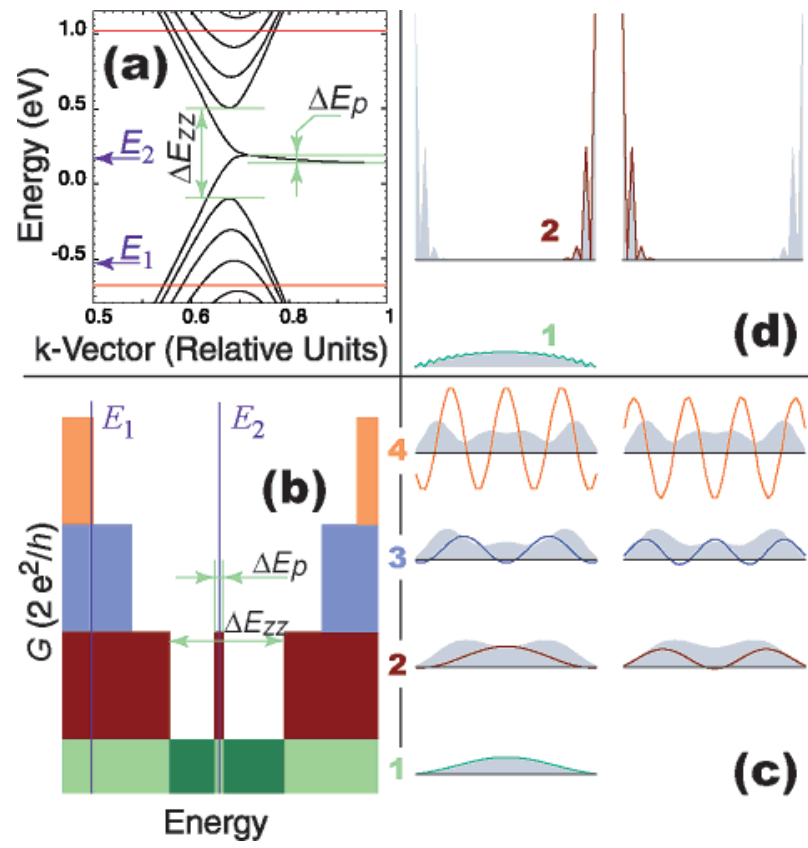

Figure 2. (a) Band-structure for zigzag-edge GNS 40-ZCs wide. (b) Conductance vs energy plot for the energy range marked in pane (a) with red lines. (c) Projected current per unit energy corresponding to energy $E_{1}$ vs lateral strip direction for separate conducting channels grouped by the band. All plots are normalized to the same relative scale. Different colors are used to indicate contributions from corresponding bands in pane (b). All bands except the first one give rise to two right moving conducting channels. Shaded regions correspond to the cumulative contribution from the all channels belonging to the same band. Dark-green regions in pane (b) mark single-channel regions. (d) Current per unit energy vs lateral strip direction for separate conducting channels corresponding to energy $E_{2}$.

low because of the small overlap between the edge and the interior states and the relatively small number of intermediate states available. Also, initially the energy lowering associated with a small increment of the spin-polarized density at the corresponding edge is proportional to the spin density already accumulated at that edge. Because the spin density of the spin-unpolarized state is zero, no first-order driving force is present to transform the system from the spin-unpolarized to the spin-polarized states within a mean field approach. Thus, even if the critical voltage is not applied permanently, the spin-unpolarized state might be maintained continuously if the time intervals between successive voltage pulses are less than the time required to return to the spin-polarized state. Hence, in the following, we assume that the applied bias is above spin-unpolarized state threshold for stability and/or the frequency is sufficiently high to prevent relaxation to the spin-polarized state. Therefore, we assume the tightbinding model that yields results for the electronic structure practically identical to corresponding spin-restricted firstprinciples calculations. ${ }^{18}$

Each zigzag-edge GNS can be thought of as a series of $N_{z z}$ parallel zigzag chains (ZCs) so that the strip width in terms of the $\mathrm{C}-\mathrm{C}$ bond distance $d(\approx 0.142 \mathrm{~nm})$ is given by $d\left(3 N_{z z}-2\right) / 2$. Thus, for example, the zigzag-edge GNS 40-ZCs wide has a width of $8.38 \mathrm{~nm}$ measured from outermost carbon atom at one edge to outermost carbon atom at the other edge. These wires have zigzag edges, which we assume are terminated by single $\mathrm{sp}^{2} \sigma$ bonds to hydrogen atoms. We also terminate the armchair-edge GNSs in the same way.

To better understand the difference between the nature of transport in zigzag- and armchair-edge GNSs, it is instructive to compute the current density contributions from each ballistic channel, assuming a two-terminal geometry with the perfect strip connecting the left and right contacts. First, all allowed $k$-vectors $\left\{k_{\mathrm{i}}\right\}$ associated with right-moving waves for a given energy $E$ are computed through the transfer matrix technique. Then wave functions and density matrix contributions $\left\{D^{n}(E)\right\}$ from every right-moving wave are obtained. The current per unit energy between orbitals $\alpha$ and $\beta$ associated with $n$th channel is given by $i_{\alpha, \beta}^{n}(E)=(4 e / \hbar) \mathrm{Im}$ $\left[H_{\alpha, \beta} D_{\alpha, \beta}^{n}(E)\right],{ }^{19}$ where $H$ is the Hamiltonian matrix. The projection of the vector sums, $i_{\alpha}^{n}(E)=1 / 2 \sum_{\beta}\left\langle i_{\alpha, \beta}^{n}(E)\right\rangle_{\mathrm{A}, \mathrm{B}}$ along the zigzag GNSs axis, averaged over A and B graphene sublattices, are plotted for different channels in Figure $2 c, d$ as a function of index $\alpha$ across the strip. The remarkable property of the first conducting channel (green curve) profile is its smooth shape with zero $i_{\alpha}^{1}(E)$ at the edges and maximum in the middle of the strip. The first channel current density profile $i_{\alpha}^{1}(E)$ has virtually the same shape in the entire energy range of Figure $2 b$ except for the energies in close proximity to the three-channel region $\Delta E_{\mathrm{p}}$. Only the first conducting channel has non-negative $i_{\alpha}^{1}(E)$ everywhere across the strip. The $i_{\alpha}^{n}(E)$ associated with other channels have regions, where current per unit energy flows to the right interchanging with the regions where current per unit energy flows to the left. The total current $i_{n}(E)=\sum_{\alpha} i_{\alpha}^{n}(E)$ for the given channel $n$ per unit energy corresponds to the integral of profile across the strip and indeed equals $2 e / h$. This current per unit energy can be viewed as the sum $i_{n}(E)=i_{n}^{+}(E)+$ $i_{n}^{-}(E)$, where $i_{n}^{+}(E)$ and $i_{n}^{-}(E)$ are contributions from the regions where current in the given channel flows to the right and to the left, respectively. For large channel index $n$, the absolute values of $i_{n}^{+}(E)$ and $i_{n}^{-}(E)$ can become fairly large, e.g., the orange channels in Figure 2c. Thus, even small relative change of $i_{n}^{ \pm}(E)$ due to the strip distortion may cause substantial degradation of $i_{n}(E)$. The closer $E$ is to the band edge, the larger are $\left|i_{n}^{+}(E)\right|$ and $\left|i_{n}^{-}(E)\right|$ and, therefore, the more sensitive the channel to defects. The sums of $i_{n}(E)$ over $n$ belonging to the same band are depicted in Figure $2 \mathrm{c}, \mathrm{d}$ by gray shades. These sums are always non-negative and quickly spread over the entire strip cross-section with increasing $n$. The higher $i_{\alpha}^{n}(E)$ near the strip edges, the more prone to degradation is the transmission due to edge defects. If more than one channel is available for the given energy, the highest $n$ channel operates as a "scattering sink" for other channels, which are coupled to channel $n$ through scattering processes. Thus, for zigzag-edge GNSs, the best ballistic transmission, i.e., the largest localization length, is obtained in the single-channel regime. The corresponding energy window is marked by a dark-green color in Figure $2 b$.

The current per unit energy $i_{\alpha}^{1}(E)$ associated with the first channel of the armchair-edge GNS is almost uniform across 
the strip. Nonzero $i_{\alpha}^{1}(E)$ at the strip edges causes strong backscattering due to edge disorder, ${ }^{11}$ which makes armchair GNS much less suitable for connecting electronic components.

Operational voltage range of the GNS circuit is restricted to the single-channel window $\Delta E_{z z}$ that has a size inversely proportional to the strip width, $W\left(\Delta E_{\mathrm{zz}} \cdot W \approx 5.3 \mathrm{eV} \mathrm{nm}\right)$ for zigzag-edge GNSs. ${ }^{11}$ Thus, if the desired voltage range is $0.75 \mathrm{~V}$, the width of the zigzag-edge GNS wires throughout the circuit should not be greater than $\approx 7.0 \mathrm{~nm}$.

Position-resolved currents and position-resolved currents per unit energy presented in Figures 3-6 are computed by spatial averaging of the current per unit energy between orbitals $\alpha$ and $\beta$

$$
i_{\alpha, \beta}(E)=(4 e / \hbar) \operatorname{Im}\left[H_{\alpha, \beta} D_{\alpha, \beta}(E)\right]
$$

Here $D(E)$ is the energy-resolved nonequilibrium density matrix evaluated through the standard real-space nonequilibrium Green's function formalism. ${ }^{19}$ The total current per unit energy through the two-terminal system is obtained by summation over all pairs of orbitals lying on different sides of an arbitrary imaginary line crossing the strip

$$
i(E) \equiv G(E) / e=\sum_{\alpha, \beta} i_{\alpha, \beta}(E)
$$

where $G(E)$ is the conductance. The total current through the two-terminal device is

$$
i=\int_{\mu_{\mathrm{R}}}^{\mu_{\mathrm{L}}} i(E) \mathrm{d} E
$$

where $\mu_{\mathrm{L}}$ and $\mu_{\mathrm{R}}\left(\mu_{\mathrm{L}}>\mu_{\mathrm{R}}\right)$ are the Fermi levels of the left and right contacts, respectively, under nonequilibrium conditions. The difference between $\mu_{\mathrm{L}}$ and $\mu_{\mathrm{R}}$ is determined by the applied voltage: $\left(\mu_{\mathrm{L}}-\mu_{\mathrm{R}}\right) / e=V_{\text {app }}$. Three terminal systems (splitters) are treated as topologically two-terminal ones with two output leads (e.g., top and bottom leads in Figure $3 b$ ) joined into a single terminal described by a selfenergy matrix

$$
\Sigma=\left(\begin{array}{ll}
\Sigma_{1} & 0 \\
0 & \Sigma_{2}
\end{array}\right)
$$

where $\Sigma_{1,2}$ are the contact self-energy matrices for separate output leads.

To minimize power loss in the interconnecting wires, one needs the means to split and turn electron flux without backscattering. Figure 3 presents the symmetric splitter, which consists of zigzag-edge GNSs of the same widths. The remarkable property of this configuration is near-zero reflection within the entire single-channel energy window with the total transmission, including both top and bottom strips, better than $99.9 \%$. Transmission deteriorates for energies that allow more than one open channel (light-yellow
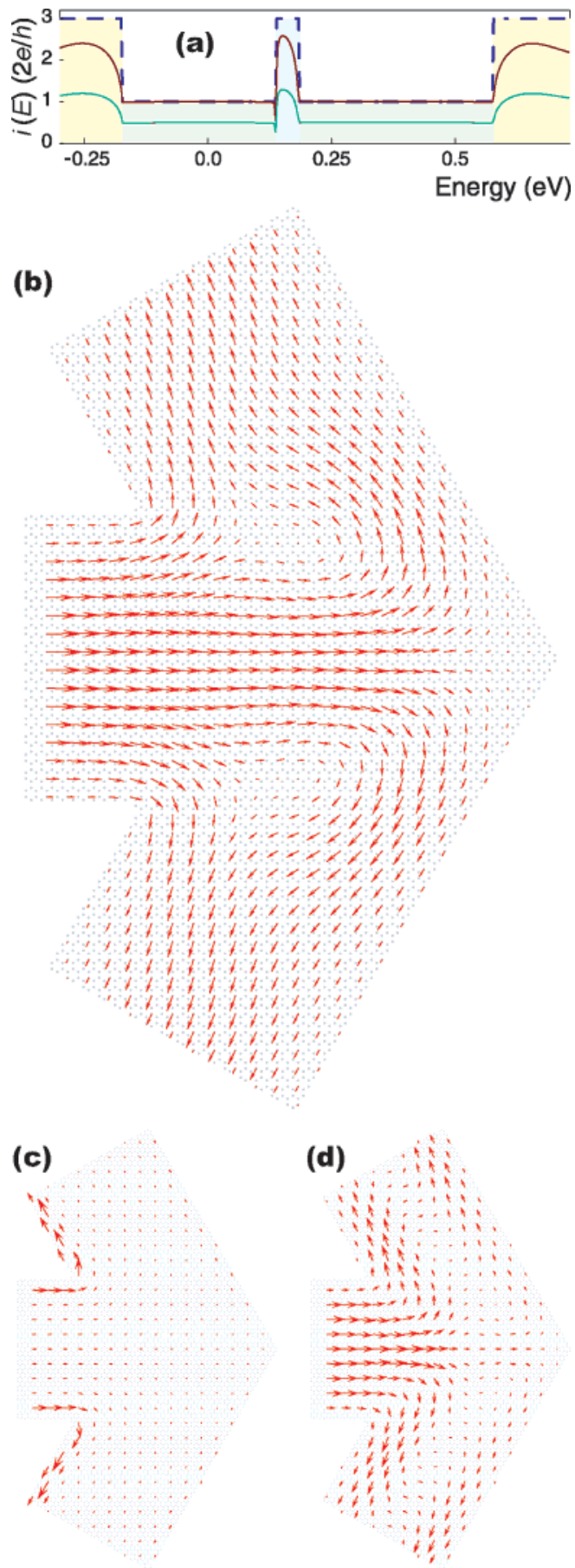

Figure 3. Symmetric splitter. (a) Splitter currents per unit energy $i(E) \equiv \sum_{n} i_{n}(E)$. Blue dashed line is $i(E)$ in the ideal zigzag-edge strips 32-ZCs wide, which constitute the splitter. Dark-red line is the sum of the $i(E)$ transmitted into both output leads. Green line is $i(E)$ transmitted into top (bottom) strip of the splitter, which is exactly $1 / 2$ from the total $i(E)$. (b) Current distribution per unit energy integrated over single-channel window (highlighted with light-green in pane (a)). (c) Current distribution per unit energy integrated over three-channel region near Fermi level (light-blue in pane (a)). (d) Current distribution per unit energy integrated over three-channel regions (light-yellow in pane (a)). Panes $(b-d)$ have different scales.

and light-blue regions in Figure 3a). Scattering from the first to the second and third conducting channels can be observed in Figure 3d. Incoming current is composed from the mix 

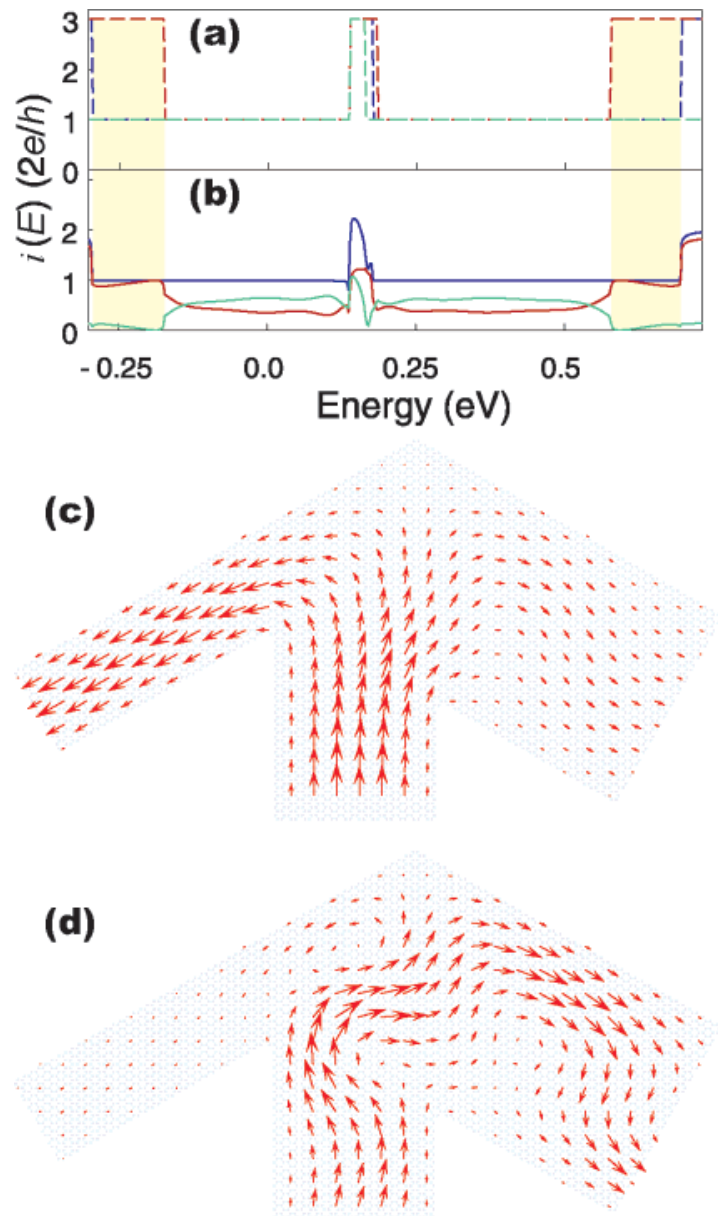

Figure 4. Asymmetric splitter. (a) $i(E)$ in the perfect zigzag-edge strips constituting the splitter: green line $-i(E)$ in the left zigzagedge GNS 14-ZCs wide; dark-red line $-i(E)$ in the right zigzagedge GNS 32-ZCs wide; blue line- $i(E)$ in the central zigzag-edge GNS 24-ZCs wide. (b) $i(E)$ in different leads of the splitter. Blue, input $i(E)$ in the central strip; green, output $i(E)$ in the left strip; dark-red, output $i(E)$ in the right strip. (c) Current distribution per unit energy integrated over the energy range where all three strips have single-channel conductance. (d) Current distribution per unit energy integrated over the energy range marked with light-yellow color. These energies correspond to single-channel conductance in the central and the left strips and three-channel conductance in the right strip.

of the first and the second bands, while the transmitted current predominantly corresponds to the second band (cf. Figure 2c).

The effects of substantial edge erosion and substrateinduced defects on the splitter performance have been tested. Only minor transmission deterioration was observed as expected based on our previous work. ${ }^{11}$ Because it will be difficult to fabricate the splitter with exactly the same strip widths, the asymmetric splitter configuration presented in Figure 4 has also been tested. Although for the asymmetric case, the currents per unit energy $i(E) \equiv \sum_{n} i_{n}(E)$ are unequally distributed between the outgoing leads, the sum of currents per unit energy in both output leads averaged over the single-channel energy range of the input strip is still $0.9982 e / h$.

An interesting behavior is observed when the wide output lead operates in the three-channel regime, while the other
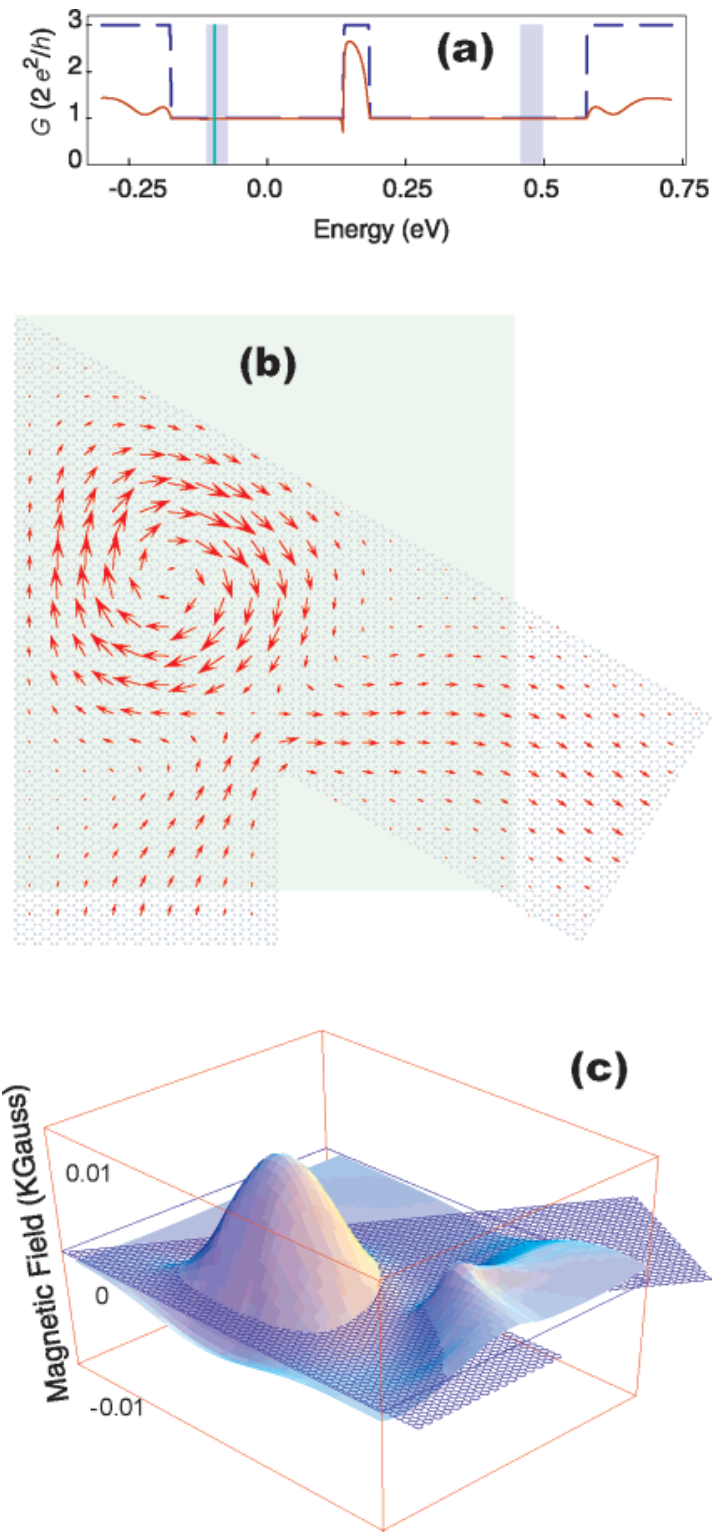

Figure 5. Symmetric $120^{\circ}$ turn composed of zigzag-edge GNSs $32-Z C s$ wide. (a) Conductances of the $120^{\circ}$ turn (dark-red) and perfect GNS 32-ZCs wide (dashed blue). Light-blue regions correspond to the energy ranges for which the circular current pattern is observed. (b) Current per unit energy pattern for the energy marked with blue vertical line in pane (a). (c) Projection of magnetic field along a normal into the page in pane (b) in the region marked with light-green in that pane integrated over the energy range corresponding to the left light-blue rectangle in pane (a).

leads operate in single-channel modes. Corresponding energies are marked with light-yellow in Figure 4a,b and are characterized by near-perfect transmission through the wide lead and near-zero transmission through of the narrow lead; the sum of the two is still better than 0.998 .

The " "'-shaped splitter is the only configuration with nearly $100 \%$ transmission we were able to find. The "T"shaped zigzag-to-armchair and armchair-to-zigzag splitters along with the "Y"-shaped zigzag-to-zigzag splitter have been also tested. For all these configurations, the backscattering in the single-channel mode is comparable to or even higher than the transmission. 

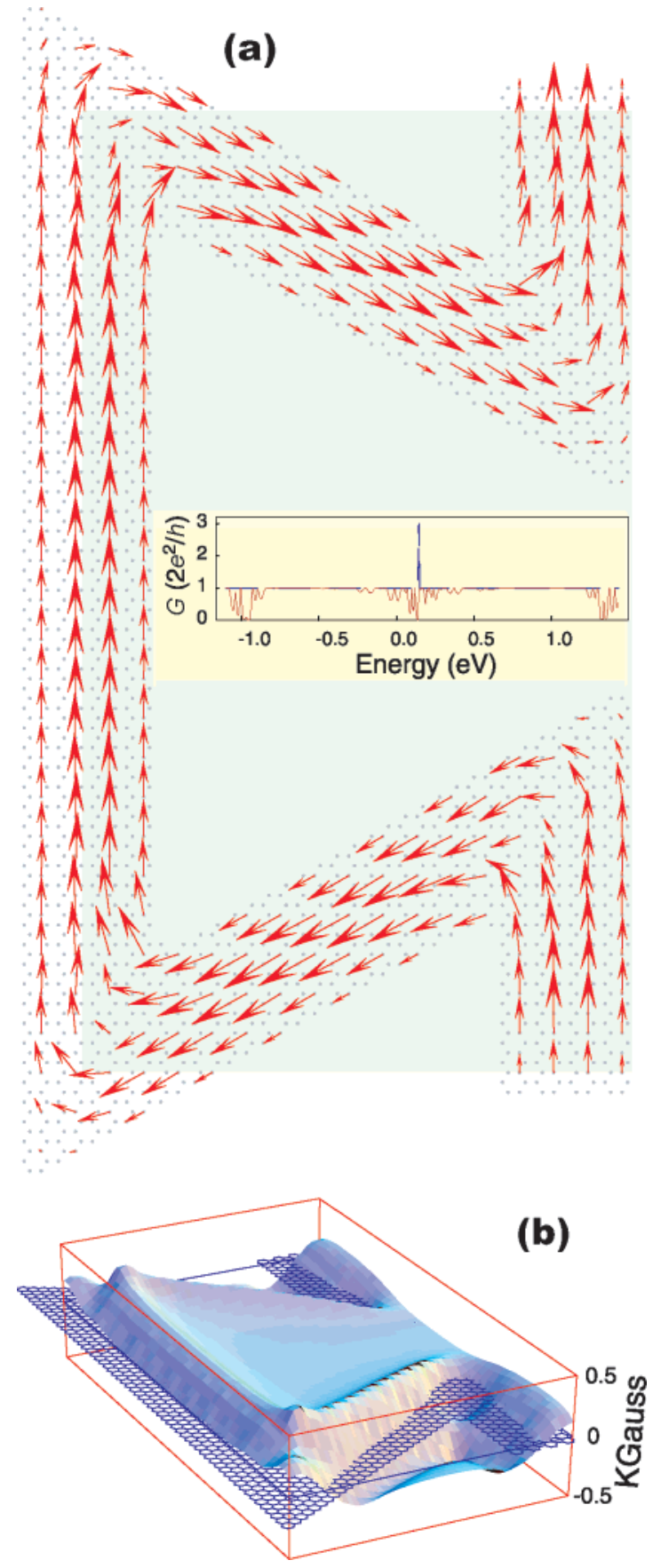

Figure 6. GNS loop composed of zigzag-edge GNSs 8-ZCs wide with four $120^{\circ}$ turns. (a) $i(E)$ integrated over single-channel window. Insert: conductance of the loop (dark-red), and conductance of the ideal zigzag-edge GNS 8-ZCs wide (dashed blue). (b) Projection of magnetic field along a normal into the page in pane (a) in the region marked with light-green in that pane.

The $120^{\circ}$ turn depicted in Figure 5 has perfect transmission inside the entire single-channel energy window. For certain energies inside this window, more than one conducting channel is locally allowed in the kink area because the "effective width" in the kink region is larger than the width of the strips composing the $120^{\circ}$ turn. That leads to interesting interference effects, which are stable to edge and substrate-induced distortions. The current per unit energy exhibits a pronounced circular pattern in the energy ranges marked by light-blue color in Figure 5a. The circular pattern

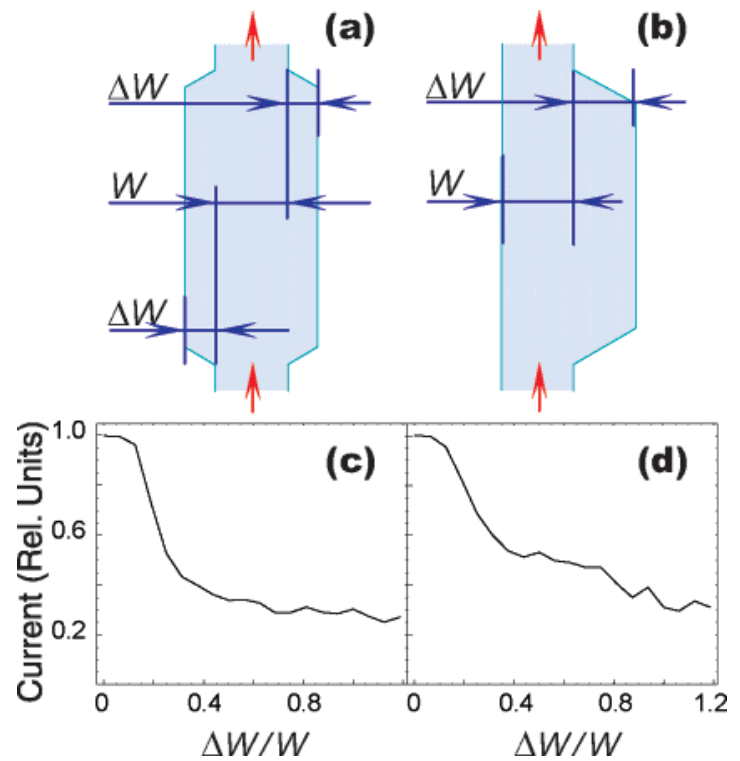

Figure 7. Effects of abrupt changes in strip width on current attenuation. (a) Symmetric and (b) asymmetric attenuator configurations. Relative integrated current as function of the relative width increase for (c) symmetric, and (d) asymmetric attenuator configurations. Current is obtained by integration over the single-channel window.

exhibits a resonant behavior, ${ }^{20}$ which yields a current per unit energy in the "resonant cavity" of substantially higher magnitude than the input/output currents per unit energy (Figure 5b). Circular currents have the same directions and similar patterns for left and right energy intervals marked with blue in Figure 5a. This circular current pattern gives rise to a weak, but highly localized magnetic field plotted in Figure 5c.

The $120^{\circ}$ turn conductance inside the single-channel window deviates slightly from $2 e^{2} / h$ for very narrow strips. The 4-turn loop composed of GNSs only 8-ZCs wide is presented in Figure 6. For this extreme example, $i(E)$ integrated over single-channel window is $88 \%$ of the ideal. The magnetic field associated with this current loop is of the order of 0.1 KGauss, which is about 5-10 times weaker than the field used to record data on conventional ferromagnetic coatings. The secondary effect of the magnetic field is negligible because, even for slow electrons with velocities $10^{6} \mathrm{~cm} / \mathrm{s}$, the cyclotron radius exceeds $1 \mu \mathrm{m}$. The inductance of the loop is of the order of $10^{-16} \mathrm{H}$ and hence the loop impedance in $\mathrm{THz}$ range is negligible.

The almost reflectionless nature of $120^{\circ}$ turn is still preserved in the asymmetric $120^{\circ}$ turns composed from the strips of different widths. The degree of reflection depends on the relative width difference and is of the order of $1 \%$ if one strip is twice as wide as the other. Such turns might serve as compact reflectionless connectors between the strips of different widths.

Abrupt variation in the strip width causes strong elastic backscattering and can be used for the controlled signal attenuation and/or for obtaining the desired voltage drop. The abrupt constriction operates similarly to a moderately nonlinear resistor. Figure 7 plots current attenuation as a 


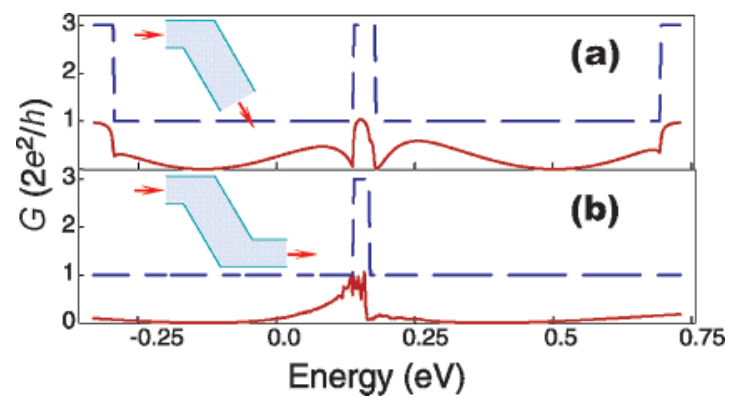

Figure 8. Conductance of the asymmetric $60^{\circ}$ turns composed of zigzag-edge GNSs 16- and 24-ZCs wide (dark-red). Inserts show the turns geometry. (a) Single turn. Dashed blue line, conductance of the ideal zigzag-edge GNS 24-ZCs wide. (b) Two $60^{\circ}$ turns connected in series. Dashed blue line, conductance of the ideal zigzag-edge GNS 16-ZCs wide.

function of the relative width increase. Plots were obtained for $W$ corresponding to GNSs 32-ZCs wide and were found to show little sensitivity to the absolute width of the strip. The sensitivity to the length variation of the widened region is also insignificant as long as the aspect ratio of the wide region is higher than 3 . The increase of the strip width beyond the plot range does not lead to the noticeable current decrease, but rather causes the irregular oscillations of $I(\Delta W / W)$.

If higher reflection is desired, then $60^{\circ}$ turns can be used. Asymmetric $60^{\circ}$ turns composed of the strips of different widths have higher reflectance than symmetric ones. Figure 8 plots the conductance of a single $60^{\circ}$ turn and two $60^{\circ}$ turns connected in series. Inside the single-channel window, the average current per unit energy is attenuated by approximately a factor of 5 by a single turn. Stronger attenuation can be obtained by serial combination of $60^{\circ}$ turns.

Strips of different widths with coinciding central axes can form reflectionless connections provided the transient region is smooth and sufficiently long. For example, if two zigzagedge GNS's, with widths that differ by a factor of 2, are connected through the transient region with the aspect ratio 10 , then the reflection is of the order of $1 \%$.

In summary, we have tested the number of patterns that can serve as the basic components in GNS circuits. They are reflectionless splitter, reflectionless $120^{\circ}$ turn, reflectionless connectors between strips of different widths and highly reflecting junctions. If high-precision electron- or ionwriting techniques are adapted for carving graphene, then all these patterns can be tested experimentally. In particular, our model indicates that conductance of the $120^{\circ}$ turn should be similar to the conductance of the straight strip and much higher than the conductance of $60^{\circ}$ turn.
Various zigzag-zigzag, armchair-armchair, and zigzagarmchair GNS intersections have also been tested. However, none of them allows transmission of the electron flux through the intersection without backscattering and splitting a substantial part of the incoming flux into the intersecting wire. We believe that the noninterfering wire crossing requires at least two graphene planes. Only few topologically simple circuits, which allow crossing-free routing, can be implemented by carving a single graphene plane. These few, however, could be used as a starting point for experimental development of graphene-based nanoelectronics.

Acknowledgment. This work was supported by ONR both directly and through the Naval Research Laboratory. We thank Daniel Gunlycke for discussions.

\section{References}

(1) Novoselov, K. S.; Geim, A. K.; Morozov, S. V.; Jiang, D.; Katsnelson, M. I.; Grigorieva, I. V.; Dubonos, S. V.; Firsov, A. A. Nature 2005, 438, 197.

(2) Zhang, Y. B.; Tan, Y. W.; Stormer, H. L.; Kim, P. Nature 2005, $438,201$.

(3) Novoselov, K. S.; Jiang, D.; Schedin, F.; Booth, T. J.; Khotkevich, V. V.; Morozov, S. V.; Geim, A. K. Proc. Natl. Acad. Sci. U.S.A 2005, 102, 10451.

(4) Berger, C.; Song, Z. M.; Li, X. B.; Wu, X. S.; Brown, N.; Naud, C.; Mayo, D.; Li, T. B.; Hass, J.; Marchenkov, A. N.; Conrad, E. H.; First, P. N.; de Heer, W. A. Science 2006, 312, 1191.

(5) Baek, I. B.; Yang, J. H.; Cho, W. J.; Ahn, C. G.; Im, K.; Lee, S. J. Vac. Sci. Technol., B 2005, 23, 3120.

(6) Bilenberg, B.; Scholer, M.; Shi, P.; Schmidt, M. S.; Boggild, P.; Fink, M.; Schuster, C.; Reuther, F.; Gruetzner, C.; Kristensen, A. J. Vac Sci. Technol., B 2006, 24, 1776.

(7) van Kan, J. A.; Bettiol, A. A.; Watt, F. Nano Lett. 2006, 6, 579

(8) Remeika, M.; Bezryadin, A. Nanotechnology 2005, 16, 1172.

(9) Nagase, T.; Gamo, K.; Kubota, T.; Mashiko, S. Microelectron. Eng. 2005, 78-79 (Special Issue), 253.

(10) Fujita, M.; Wakabayashi, K.; Nakada, K.; Kusakabe, K. J. Phys. Soc. Jpn. 1996, 65, 1920.

(11) Areshkin, D. A.; Gunlycke, D.; White, C. T. Nano Lett. 2007, 7, 204.

(12) Gunlycke, D.; Lawler, H. M.; White, C. T. Phys. Rev. B 2007, 75, 085418.

(13) Han, M.Y.; Oezyilmaz, B.; Zhang, Y.; Kim, P. Phys. Rev. Lett. 2007, 98, 206805.

(14) Chen, Z; Lin, Y.-M.; Rooks, M.J.; Avouris, P. Physica E 2007, in press, http://dx.doi.org/10.1016/j.physe.2007.06.020.

(15) Pisani, L.; Chan, J. A.; Montanari, B.; Harrison, N. M. Phys. Rev. B 2007, 75, 064418

(16) Son, Y.-W.; Cohen, M. L.; Louie, S. G. Phys. Rev. Lett. 2006, 97, 216803.

(17) White, C. T.; Li, J.; Gunlycke, D.; Mintmire, J. W. Nano Lett. 2007, 7, 825 .

(18) Miyamoto, Y.; Nakada, K.; Fujita, M, Phys. Rev. B 1999, 59, 9858.

(19) Todorov, T. N. J. Phys.: Condens. Matter 2002, 14, 3049.

(20) Areshkin, D. A.; Shenderova, O. A.; Schall, J. D.; Adiga, S. P.; Brenner, D. W. J. Phys.: Condens. Matter 2004, 16, 6851. 